\title{
Ensinando com jogos ou jogando com o ensino: a visão da comunidade brasileira de Informática na Educação sobre jogos no ensino de matemática
}

\author{
Leônidas de Oliveira Brandão ${ }^{1}$, Igor Moreira Félix ${ }^{1}$, \\ Anarosa Alves Franco Brandão ${ }^{2}$, Patricia Alves Pereira ${ }^{3}$ \\ ${ }^{1}$ Instituto de Matemática e Estatística - Universidade de São Paulo (USP) \\ São Paulo - SP - Brasil \\ ${ }^{2}$ Escola Politécnica - Universidade de São Paulo (USP) \\ São Paulo - SP - Brasil \\ ${ }^{3}$ Ibmec São Paulo \\ São Paulo - SP - Brasil \\ leo@ime.usp.br, igormfeime.usp.br, \\ anarosa.brandao@poli.usp.br, patricia.pereira@ibmec.edu.br
}

\begin{abstract}
The use of educational games has gaining special attention in the different areas of knowledge. Among the reasons why, educational games has been used due to their ludic aspects, which may motivate the learners' engagement. This work presents a research performed within the repository of the Brazilian Community of Informatics in Education. It was identified almost six hundred papers using games in education in the last ten years. However, most of them focused on the artifact presentation. Only eleven papers analysed effective learning associated with using educational games in mathematicss were identified.
\end{abstract}

Resumo. A utilização de jogos educacionais vem recebendo especial atenção nas diversas áreas do conhecimento. Dentre as razões para seu emprego no apoio à educação destaca-se o aspecto lúdico, que pode motivar o aprendiz a se engajar nas atividades didáticas. Neste trabalho é relatada uma pesquisa realizada nas publicações da comunidade de Informática na Educação, nos últimos dez anos, que identificou quase seiscentos trabalhos utilizando jogos digitais na educação. Entretanto, a ampla maioria concentra-se na apresentação de artefatos, tendo sido identificados apenas onze artigos que analisaram a aprendizagem decorrente do uso da ferramenta no ensino de matemática.

\section{Introdução}

A literatura indica que o aprendizado de matemática apresenta dificuldades nos variados níveis escolares. Por exemplo, [Weber 2001] cita que estudantes do ensino superior apresentam grande dificuldade com a linguagem matemática. Da mesma forma, podemos encontrar variados estudos relatando dificuldades no processo de ensino-aprendizagem de conteúdos relacionados à matemática no ensino fundamenta.

Em particular, no Brasil os dados do IDEB (Índice de Desenvolvimento da Educação Básica) e do PISA (Programme for International Student Assess- 
VII Congresso Brasileiro de Informática na Educação (CBIE 2018)

Anais do XXIX Simpósio Brasileiro de Informática na Educação (SBIE 2018)

ment) indicam que o desempenho dos estudantes em matemática é bastante deficiente [Pereira and Brandão 2009, Reis and Silveira 2011]. Parte das deficiências com matemática no ensino superior pode ser explicada por falhas no ensino fundamental, principalmente devido à característica construtiva do conhecimento em matemática, de modo que para a sedimentação de novos conhecimentos, é necessário o domínio de seus fundamentos.

Nesse contexto, nota-se um grande esforço para criar métodos, técnicas e software para apoiar esse processo. Uma linha em particular é o emprego de jogos, devido principalmente aos seus aspectos lúdicos, retroação rápida sobre as ações de usuários e a organização de níveis crescentes de desafios [Marne et al. 2012, Cardoso et al. 2013].

Nesse artigo é relatada uma ampla pesquisa realizada na comunidade de Informática na Educação, buscando os trabalhos envolvendo o uso de jogo digital na educação relacionada com a matemática. Examinamos a Revista Brasileira de Informática na Educação, os anais do Simpósio Brasileira de Informática na Educação e do Workshop de Informática na Escola e os demais eventos da CEIE (Comissão Especial de Informática na Educação entre 2008 e 2017.

Os principais achados dessa pesquisa estão aqui apresentados, notadamente o crescimento quase linear sobre o uso de jogos na educação quando são consideradas todas as áreas do conhecimento, contra uma estagnação quado se considera a matemática.

O artigo está organizado da seguinte forma: a seção 2 descreve a metodologia da revisão sistemática, seus protocolos e a execução do processo de seleção; enquanto a seção 3 apresenta os resultados em análises quantitativas e qualitativas, além de responder às questões de pesquisa; e por fim, as considerações finais são dissertadas na seção 4.

\section{Metodologia da Revisão Sistemática}

Nesta seção é apresentada a metodologia da Revisão Sistemática (RS), realizada a partir de questões exploratórias, com o objetivo de identificar evidências relevantes para fundamentar e avaliar criticamente a validade do estudo proposto neste artigo [Kitchenham 2004]. Para este estudo foi elaborada uma questão de âmbito geral, utilizada para nortear a coleta dos estudos primários, com o objetivo de investigar a eficácia do uso de jogos para o ensino de matemática. A seguir são apresentados: o protocolo adotado, as análises quantitativas e qualitativas e os resultados da RS.

\subsection{Protocolo}

Para orientar a coleta de evidências, a RS foi guiada pelo seguinte protocolo: (i) definição da questão principal e das subquestões de pesquisa; (ii) definição das Strings de busca; (iii) elaboração e aplicação das estratégias de pesquisa; (iv) definição do processo de seleção; e, (v) extração e apuração dos dados.

Para este estudo foi elaborada a seguinte questão principal de pesquisa: "Quais artigos publicados pela comunidade brasileira de Informática na Educação que envolvem o tema de jogo digital no ensino da Matemática investigam a efetividade do aprendizado apoiado com este recurso?". Seis questões decorrentes foram utilizadas para ajudar a responder a questão principal, listadas a seguir:

- QP1. Foram utilizados métodos para medir a eficácia didática? Se sim, quais? 
VII Congresso Brasileiro de Informática na Educação (CBIE 2018)

Anais do XXIX Simpósio Brasileiro de Informática na Educação (SBIE 2018)

- QP2. Quais os assuntos/tópicos da Matemática são abordados nos jogos?

- QP3. A qual nível/grau (fundamental, médio ou superior) o jogo é destinado?

- QP4. Foi detectado algum benefício de se empregar os jogos no ensino de Matemática?

- QP5. Foram detectados problemas de se utilizar jogos educacionais?

- QP6. Quais dos trabalhos desenvolveram jogo próprio e quais aplicaram jogos já desenvolvidos?

A partir da questão principal e das demais, foi construída uma String de busca abrangente, que contemplasse os artigos publicados tanto em português quanto em inglês: "jogo OR game".

Como o interesse deste estudo é avaliar como a comunidade brasileira de Informática na Educação aborda a efetividade no uso de jogo digital no ensino de Matemática, a base de conhecimento usada para realizar a busca foi o repositório de publicações da Comissão Especial de Informática na Educação (CEIE) da Sociedade Brasileira de Computação (SBC). Este repositório engloba as publicações de: Simpósio Brasileiro de Informática na Educação (SBIE); Workshop de Informática na Escola (WIE); Workshops do Congresso Brasileiro de Informática na Educação (WCBIE) e a Revista Brasileira de Informática na Educação (RBIE).

As publicações da CEIE são de acesso livre e encontram-se disponíveis a partir de seu portal, sendo possível realizar pesquisas personalizadas por meio dos mecanismos de busca oferecidos nos sítios. Contudo, a fim de executar buscas mais refinadas, optou-se em conduzir uma mineração de textos em ambiente local. Para este procedimento, foram coletadas as publicações referentes ao período de 2008 a 2017, diretamente dos portais de anais do SBIE, WIE, WCBIE e da RBIE, totalizando 2.909 arquivos.

O processo de mineração de textos foi realizado com a ferramenta RapidMiner $^{1}$, instalada com a extensão “Text Mining Extension”, para possibilitar o pre-processamento dos textos antes da realização efetiva da busca. A figura 1 ilustra o processo realizado nessa mineração:

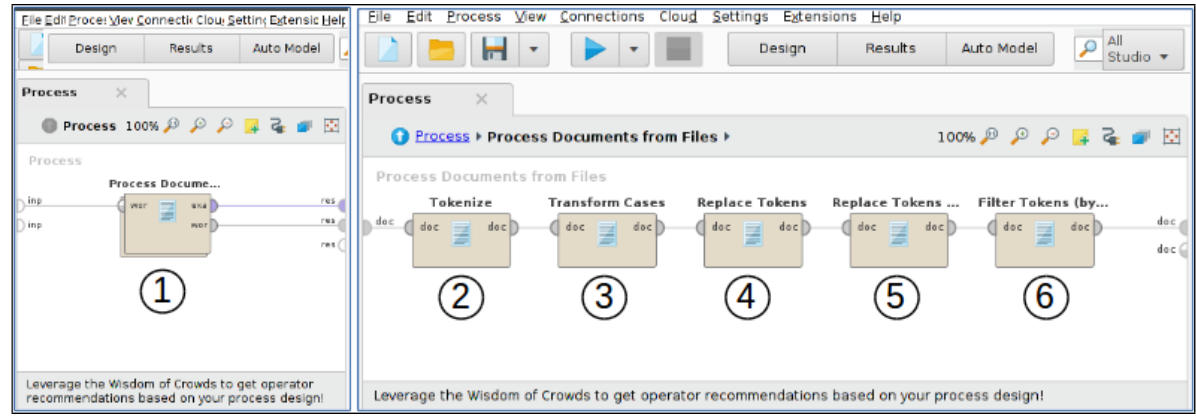

Figura 1. Processo de mineração de textos no RapidMiner

O processo é iniciado pelo Process Documents from Files (1), que gera um conjunto de vetores do conteúdo textual de cada um dos 2.909 arquivos. Estes são processados individualmente pelos operadores listados a seguir: Tokenize (2): divide o texto em um conjunto de palavras; Transform Cases (3): converte todas as palavras para minúsculo;

\footnotetext{
${ }^{1}$ RapidMiner: disponível em http://rapidminer.com/. Acessado em 20/05/2018.
} 
VII Congresso Brasileiro de Informática na Educação (CBIE 2018)

Anais do XXIX Simpósio Brasileiro de Informática na Educação (SBIE 2018)

Replace Tokens (4): substitui as palavras consideradas sinônimas: "jogo", “jogos" e "games" foram substituídas por "game"; Replace Tokens (5): substitui as palavras com caracteres especiais: "game.", "game,", "game!” e "game?" foram substituídas por "game”. Filter Tokens (by Content) (6): executa a busca adotando como critério o filtro de palavras iguais a "game". Ao final do processo de mineração das 2.909 publicações, foram apontadas 1.190, nas quais a palavra "game" é encontrada em seu conteúdo.

O processo de seleção da RS começa pela definição dos critérios de exclusão das publicações retornadas após a mineração dos textos (Tabela 1). Como a revisão sistemática tem como foco os reflexos da utilização de jogos educacionais no ensino da matemática, os critérios envolveram regras que elegessem publicações que apresentam algum impacto da utilização destes artefatos no aprendizado.

Tabela 1. Critérios de exclusão

\begin{tabular}{|l|l|}
\hline ID & \multicolumn{1}{|c|}{ Descrição } \\
\hline E1 & Publicações que abordam jogos, mas não focam em sua utilização \\
\hline E2 & Publicações que não abordam os jogos para o ensino \\
\hline E3 & Publicações que não tratam do ensino de tópicos da matemática \\
\hline E4 & Publicações que não respondem às questões de pesquisa \\
\hline
\end{tabular}

O processo de seleção das publicações é apresentado na figura 2. Dentro deste processo, no primeiro momento foi realizada uma filtragem nos resultados retornados pelo RapidMiner, a fim de identificar artigos que apesar de terem sido selecionados, não apresentavam a ocorrência das palavras-chave ( 8 artigos). Ainda na filtragem, por meio da leitura do título e do resumo, foram descartadas descartadas aquelas publicações que estavam fora do contexto analisado (635 artigos).

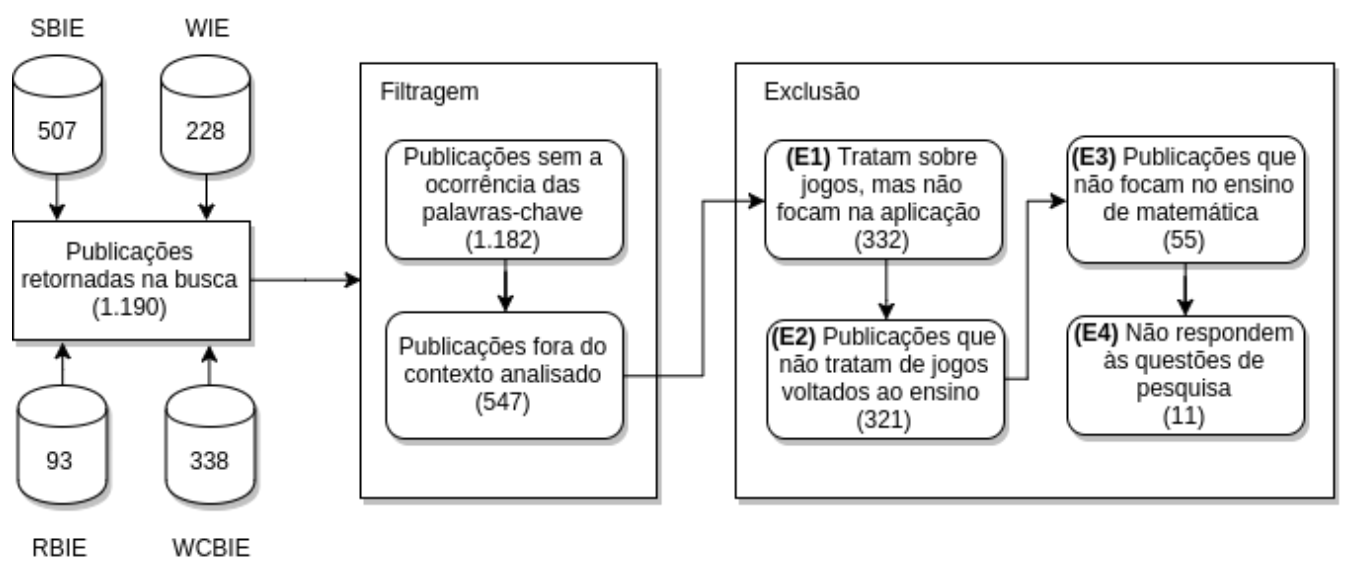

Figura 2. Execução do processo de seleção das publicações

Na fase de exclusão, 215 artigos que abordam a temática de jogos, sem focar em sua aplicação foram descartados (E1). Enquanto outros 11 trabalhos foram removidos por não serem voltados ao ensino (E2). E 266 por não tratar do ensino de algum tópico da matemática (E3), restando 55 trabalhos. Estes 55 passaram por uma leitura completa, a fim de encontrar as respostas às questões de pesquisa. Sendo que ao fim da leitura, 44 foram descartados por não responderem às perguntas $(\mathbf{E 4})$, resultando em 11 publicações para inclusão e extração de dados. 
VII Congresso Brasileiro de Informática na Educação (CBIE 2018)

Anais do XXIX Simpósio Brasileiro de Informática na Educação (SBIE 2018)

Algumas ameaças à validade da revisão sistemática devem ser consideradas. A primeira é a restrição do período das publicações, de 2008 a 2017. Uma vez que podem existir estudos relevantes não incluídos por estarem fora desse interstício. Outra ameaça que merece destaque é a formulação das palavras de busca, afinal podem existir termos sinônimos desconsiderados.

\section{Resultados}

\subsection{Análise quantitativa}

Os dados quantitativos apresentados fornecem informações relevantes sobre a situação da pesquisa da comunidade brasileira de Informática na Educação no uso de jogos no processo de ensino e aprendizagem. Do total das 2.909 publicações analisadas, no período entre 2008 e 2017, apenas 321 (11,03\%) tratam do uso efetivo de jogos para o ensino, sendo que destes, apenas $55(1,89 \%)$ para o ensino de matemática. Quando consideramos jogos para o ensino de matemática, apenas $4(0,14 \%)$ deles abordam jogos inclusivos, sendo dois para deficiência visual [Dantas et al. 2013, Macedo et al. 2017], um para deficiência cognitiva [Silva et al. 2011] e um para portadores de Síndrome de Down [Lundgren and Felix 2017].

Contudo, vale destacar a tendência de crescimento no interesse em estudos sobre jogos educacionais, conforme evolução apresentada no gráfico da figura 3. Apesar disso, o interesse em jogos para o ensino de matemática apresentou crescimento pouco significativo na última década.

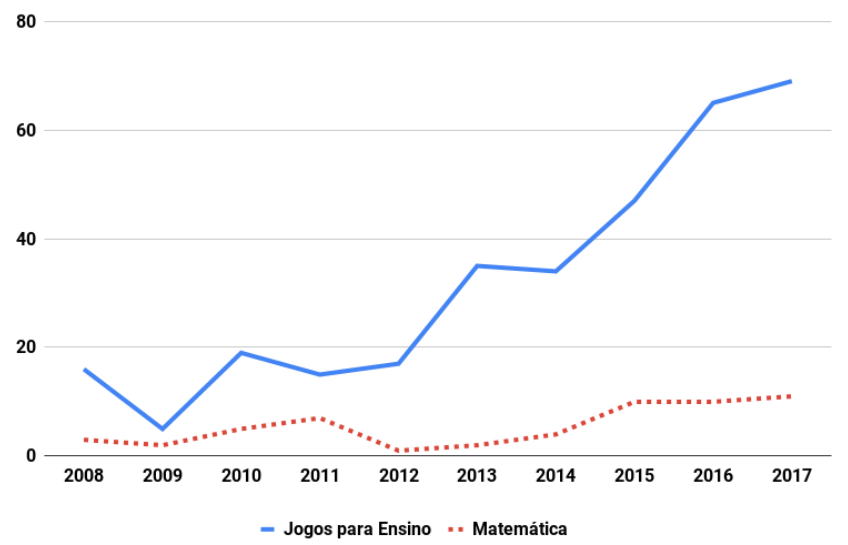

\section{Figura 3. Evolução das publicações da CEIE sobre jogos para ensino em geral e para matemática}

Outra característica observada refere-se à fonte dos jogos para o ensino de matemática, que foram apresentados nas 55 publicações analisadas: 22\% eram jogos de terceiros e $78 \%$ jogos próprios. Tal fato pode indicar carência de jogos de matemática disponíveis no mercado e que atendam às necessidades de ensino, exigindo que os pesquisadores desenvolvam seus próprios artefatos antes de conduzir seus estudos. A distribuição dos jogos por nível de ensino pode ser observada no gráfico da figura 4, sendo a maior parte deles voltados às séries iniciais do Ensino Fundamental I (45,8\%). 
VII Congresso Brasileiro de Informática na Educação (CBIE 2018)

Anais do XXIX Simpósio Brasileiro de Informática na Educação (SBIE 2018)

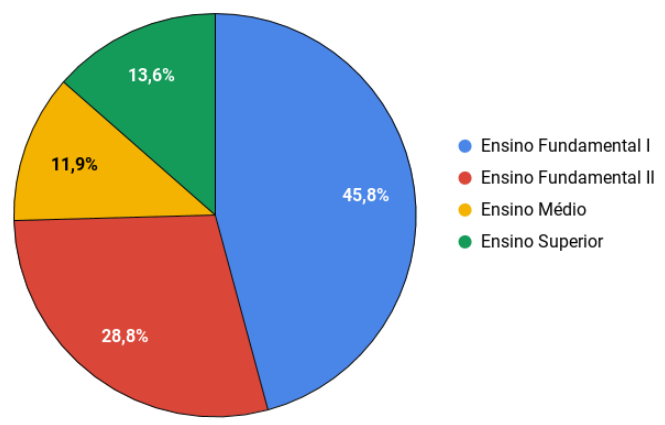

Figura 4. Distribuição por grau de ensino dos jogos para matemática

Quanto à origem geográfica desses estudos, percebe-se que as regiões brasileiras com mais produções sobre jogos para o ensino de matemática é a Sudeste (38,2\%), seguida pelo Nordeste $(34,5 \%)$. E aquela com menos estudos a respeito é a Região Norte $(1,8 \%)$. As regiões Sul e Centro Oeste apresentaram, respectivamente, 21,8\% e 3,6\% dos artigos selecionados.

Outra observação interessante refere-se à origem das publicações dentro do repositório da CEIE. O gráfico da figura 5, apresenta a distribuição dos artigos selecionados. A maior parte das contribuições foram divulgadas no SBIE, sendo 24 artigos sobre jogos de matemática, dos quais 8 apresentaram experimentos sobre o uso efetivo de jogos no processo de ensino e aprendizagem. Nos demais nota-se que a maioria apenas apresentou o jogo mas não seus reflexos efetivos no ensino. A RBIE apresentou menor participação para esse estudo.

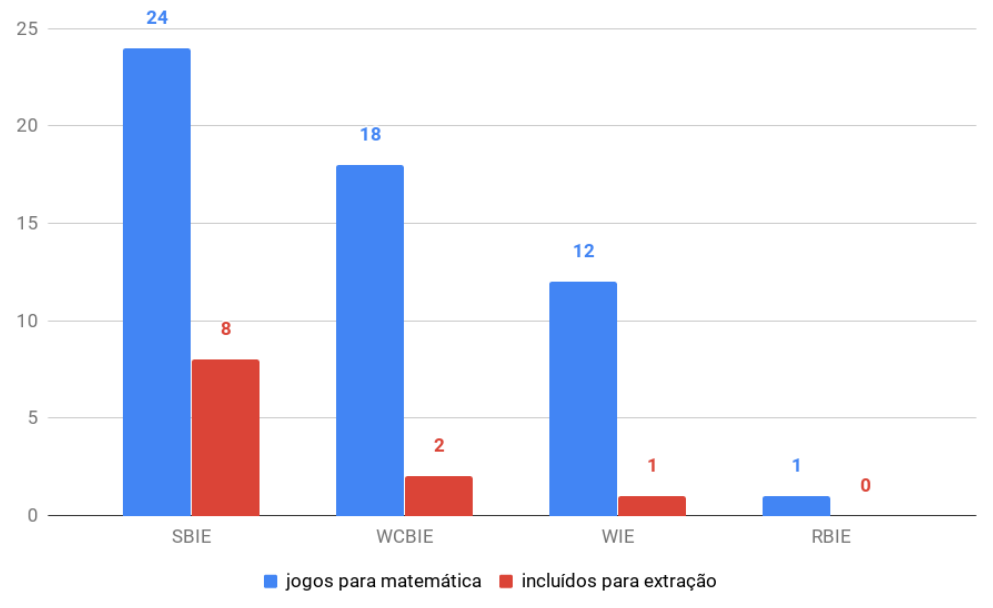

Figura 5. Artigos para matemática e incluídos na extração, de acordo com o periódico

\subsection{Análise qualitativa}

As análises das informações extraídas das publicações abriram espaço para a interpretação das percepções e entendimento sobre a situação da efetividade do uso de jogos no processo de ensino de matemática. Uma síntese dessa análise, individual por publicação, é apresentada a seguir. 
VII Congresso Brasileiro de Informática na Educação (CBIE 2018)

Anais do XXIX Simpósio Brasileiro de Informática na Educação (SBIE 2018)

[Gomes et al. 2008] usa o jogo KickRobot, que simula arremessos em uma partida de basquete, para ensinar conceitos que envolvem matemática e física. A avaliação foi feita com o uso de questionários aplicados no início e final do experimento e foi observado maior engajamento e participação dos alunos nas atividades. Neto e colegas [2011] relataram estudo que aplica o uso de jogos para treinamento de professores de escolas com baixo índice no IDEB de 2007, como forma de criar meios para que os professores motivassem os alunos a aprender e, consequentemente, melhorar os índices. Quando comparados com 2007, os índices de 2009 indicaram melhoras em todas as escolas.

O uso de jogos para a consolidação da multiplicação com cálculo mental foi adotado por [Cardoso et al. 2013]. Os alunos tinham restrição de tempo para apresentar a resposta calculada mentalmente e, através de questionários, avaliaram maior motivação com o uso do jogo, apesar de apresentarem desempenho levemente inferior se comparado ao uso de dedos ou riscos no papel para auxiliar o cálculo. O uso de jogos para apoiar o ensino de axiomas de geometria foi avaliado como positivo no que diz respeito ao desempenho dos alunos nos testes aplicados, conforme relatado por [Neto et al. 2015b]. Ainda sobre ensino de geometria e aritmética, [de Araújo et al. 2016] fez uso de dispositivos eletrônicos de baixo custo para apoiar o ensino de direção, espaço, grandezas e operações aritméticas elementares no ensino fundamental. O uso de pré e pós testes avaliou como positiva a experiência. Continuando no tema aritmética para ensino fundamental, [Reis et al. 2016] descrevem experiência positiva na aprendizagem da soma usando jogos colaborativos, avaliada também com pré e pós testes.

O impacto do uso de jogos na aprendizagem de conjuntos e funções de primeiro grau foi avaliado por [Neto et al. 2015a], com alunos do ensino médio usando uma plataforma online. Os autores relatam melhora significativa no desempenho dos alunos. O impacto positivo no aprendizado de ângulos a partir do uso de dois jogos é relatado por [Castro et al. 2016]. O desenvolvimento e avaliação de uso de um jogo para melhorar o aprendizado de resolução de problemas matemáticos é relatado por [Rodrigues et al. 2017]. Neste trabalho foi avaliado o nível de aprendizagem em relação ao tempo de exposição ao jogo.

Pereira e colegas [2017] descrevem o uso de um aplicativo de de visualização de superfícies quádricas para apoiar a aprendizagem do Cálculo. Uma comparação de aprendizado com e sem o uso do aplicativo indicou leve vantagem para o aprendizado usando o aplicativo. O ensino e a aprendizagem de numerais, sequências numéricas e conjuntos para crianças e jovens portadores da Síndrome de Down é o foco de [Lundgren and Felix 2017]. Avaliação realizada usando pré e pós testes indicaram que o aprimoramento médio nas capacidades matemáticas dos participantes aumentou em $36,24 \%$.

\subsection{Repostas às questões de pesquisa}

QP1. Foram utilizados métodos para medir a eficácia didática? Se sim, quais?

Os 11 trabalhos selecionados responderam RQ1 positivamente. A tabela 2 apresenta as metodologias utilizadas para verificar o aprendizado dos estudantes após a utilização de jogos educacionais para ensino de matemática. A grande maioria dos trabalhos (7) têm empregado o uso de pré e pós-teste.

QP2. Quais os assuntos/tópicos da Matemática são abordados nos jogos? 
VII Congresso Brasileiro de Informática na Educação (CBIE 2018)

Anais do XXIX Simpósio Brasileiro de Informática na Educação (SBIE 2018)

Tabela 2. Metodologia para avaliação da aprendizagem

\begin{tabular}{|l|l|}
\hline Metodologia & Trabalhos \\
\hline \multirow{3}{*}{ Aplicação de pré e pós-teste } & [Gomes et al. 2008], [Cardoso et al. 2013], \\
& $\begin{array}{l}\text { [Neto et al. 2015b], [de Araújo et al. 2016], } \\
\text { [Reis et al. 2016], [Lundgren and Felix 2017] } \\
\text { e [Rodrigues et al. 2017] }\end{array}$ \\
\hline Análise dos resultados do IDEB & [Neto et al. 2011] \\
\hline Análise de avaliações periódicas & [Neto et al. 2015a] \\
\hline Análise dos resultados bimestrais & [Castro et al. 2016] \\
\hline Grupo experimental e de controle & [Pereira et al. 2017] \\
\hline
\end{tabular}

Dos trabalhos incluídos na revisão sistemática, existe uma variedade dos tópicos da disciplina de matemática abordados nos jogos, apesar de que cinco dos onze são voltados ao ensino de aritmética [Cardoso et al. 2013], [Reis et al. 2016], [de Araújo et al. 2016], [Lundgren and Felix 2017], [Rodrigues et al. 2017]. Os demais, envolviam trigonometria [Gomes et al. 2008], treinamento de professores [Neto et al. 2011], conjuntos e funções [Neto et al. 2015a], geometria [Neto et al. 2015b], lógica computacional [Castro et al. 2016] e cálculo [Pereira et al. 2017].

QP3. A qual nível/grau (fundamental, médio ou superior) o jogo é destinado?

Entre as publicações incluídas, a verificação da aprendizagem dos estudantes submetidos à utilização de jogos têm sido usada em todos os níveis, a saber: ensino fundamental I [Reis et al. 2016], [de Araújo et al. 2016], [Lundgren and Felix 2017], [Rodrigues et al. 2017], ensino fundamental II [Cardoso et al. 2013], [Neto et al. 2015b], [Castro et al. 2016], [Reis et al. 2016], ensino médio [Gomes et al. 2008], [Neto et al. 2015a] e ensino superior [Neto et al. 2011], [Pereira et al. 2017].

QP4. Foi detectado algum benefício de se empregar os jogos no ensino de Matemática?

[Gomes et al. 2008] observou que os estudantes passaram a interagir mais durante as aulas, com mais curiosidade e questionamento a respeito dos assuntos tratados nos experimentos práticos de robótica. O autor observa ainda, que a utilização de um simulador de robótica permite a inclusão dos estudantes com reduzidos recursos financeiros.

QP5. Foram detectados problemas de se utilizar jogos educacionais?

Não foram descritos problemas.

QP6. Quais dos trabalhos desenvolveram jogo próprio e quais aplicaram jogos já desenvolvidos?

Desenvolveram o próprio jogo: [Cardoso et al. 2013], [Neto et al. 2015a], [Neto et al. 2015b], [de Araújo et al. 2016], [Pereira et al. 2017], [Lundgren and Felix 2017], [Rodrigues et al. 2017]. Utilizaram jogos de terceiros: [Gomes et al. 2008], [Neto et al. 2011], [Reis et al. 2016], [Castro et al. 2016].

\section{Conclusões}

O uso de jogos educacionais tem ganhado relevância nos últimos anos, destacando-se como principal fonte de sucesso a motivação que desperta, principalmente, em jovens 
VII Congresso Brasileiro de Informática na Educação (CBIE 2018)

Anais do XXIX Simpósio Brasileiro de Informática na Educação (SBIE 2018)

aprendizes. Existem várias linhas de trabalho, uma dessas é a ludificação (que em Inglês recebe o nome de gamification), que adota dinâmicas típicas de jogos em outras atividades, como por exemplo, o uso de competições, rankings, prêmios ou distribuição de distintivos.

Outra linha é a dos jogos sérios e essa merece maior destaque por focar prioritariamente no aprendizado e não apenas na diversão [Marne et al. 2012]. Mas essa linha tem sua origem em atividades de treinamento, como capacitação de pilotos, e ainda recebe algumas críticas por sua seriedade, divertindo pouco [Susi et al. 2007].

A partir da base de publicações associadas à comunidade brasileira de Informática na Educação, foram identificados 547 artigos envolvendo jogos, desses 55 eram destinados ao aprendizado de algum tópico de matemática.

O resultado observado mais importante é que dos 547 artigos envolvendo jogos, 332 tinham preocupação educativa explicita, mas apenas 11 deles buscaram de alguma forma analisar se o jogador aprendeu algum conceito matemático.

Outro resultado interessante é que no intervalo de 10 anos pesquisado, o número de trabalhos envolvendo jogos cresceu significativamente (de quase 20 em 2008 para quase 80 em 2017), mas quando o foco é a matemática o número de trabalhos praticamente manteve-se constante (em torno de 11).

\section{Referências}

Cardoso, A., Giraldello, A. G., and Nalva Ap M, B. (2013). Tabuada legal: um jogo sério para o ensino de multiplicações. In Simpósio Brasileiro de Informática na EducaçãoSBIE, volume 24, page 376.

Castro, M. C., Werneck, V., and Gouvea, N. (2016). Ensino de matemática através de algoritmos utilizando jogos para alunos do ensino fundamental ii. In Anais dos Workshops do Congresso Brasileiro de Informática na Educação, volume 5, page 1039.

Dantas, A. L. P., Pinto, G. R. R., and Sena, C. P. P. (2013). Apresentando o bem: Um objeto de aprendizagem para mediar o processo educacional de crianças com deficiência visual e videntes nas operações básicas de matemática. In Simpósio Brasileiro de Informática na Educação-SBIE, volume 24, page 437.

de Araújo, J. P. P., Costa, G., and Júnior, J. G. R. (2016). Matematech: Plataforma de apoio à aprendizagem de matemática nos anos iniciais do ensino fundamental. In Simpósio Brasileiro de Informática na Educação-SBIE, volume 27, page 110.

Gomes, M. C., Barone, D. A. C., and Olivo, U. (2008). Kickrobot: Inclusão digital através da robótica em escolas públicas do rio grande do sul. In Simpósio Brasileiro de Informática na Educação-SBIE, volume 1, pages 410-419.

Kitchenham, B. (2004). Procedures for performing systematic reviews. In Keele, UK, Keele University, volume 33, page 2004.

Lundgren, A. and Felix, Z. (2017). Plataforma sam: a gamificação e a colaboração em uma plataforma de aprendizagem para o ensino da matemática em crianças portadoras de síndrome de down. In Simpósio Brasileiro de Informática na Educação-SBIE, volume 28, page 625 . 
VII Congresso Brasileiro de Informática na Educação (CBIE 2018)

Anais do XXIX Simpósio Brasileiro de Informática na Educação (SBIE 2018)

Macedo, R., Pereira, C. P., Fernandes, A., Santana, K., and Santos, A. J. d. O. S. (2017). Blinds, education and mathematics: objeto de aprendizagem sobre as operações básicas da matemática com o uso dos recursos de síntese e reconhecimento de voz. In Simpósio Brasileiro de Informática na Educação-SBIE, volume 28, page 445.

Marne, Bertrand, W. J., Huynh-Kim-Bang, B., and Labat, J.-M. (2012). The six facets of serious game design: a methodology enhanced by our design pattern library. In Proceeding of European Conference on Technology Enhanced Learning, pages 208221. Springer, Berlin, Heidelberg.

Neto, A., da Silva, A. P., and Bittencourt, I. I. (2015a). Uma análise do impacto da utilização de técnicas de gamificação como estratégia didática no aprendizado dos alunos. In Simpósio Brasileiro de Informática na Educação-SBIE, volume 26, page 667.

Neto, J. C., Bernardelli, M. S., Pessoa, M. P., Guilhem, M. S., Malucelli, A., and Reinehr, S. (2011). O uso das tic na formação de professores de escolas que obtiveram baixo ideb. In Simpósio Brasileiro de Informática na Educação-SBIE, volume 1.

Neto, S., Galdino, E., Ferreira, A., Oliveira, W., de Souza, A. A., de Oliveira, M. L. S., and Balieiro, A. (2015b). Avaliação do jogo educativo mundo de euclides: Uma abordagem multi-perspectiva. In Anais dos Workshops do Congresso Brasileiro de Informática na Educação, volume 4, page 1114.

Pereira, L., Oliveira, D., Couto, I., Oliveira, A., and da Silva, R. L. d. S. (2017). Uma ferramenta de apoio ao ensino de cálculo com realidade aumentada. In Simpósio Brasileiro de Informática na Educação-SBIE, volume 28, page 595.

Pereira, P. A. and Brandão, L. d. O. (2009). Tarefa interativa: uma proposta flexível de interatividade para o moodle. In Anais do Simpósio Brasileiro de Informática na Educação-SBIE. SBC.

Reis, L. C. and Silveira, I. F. (2011). Aproximando nativos e imigrantes digitais em sala de aula com o uso efetivo das tecnologias: uma análise contextualizada do estado de são paulo à luz do ideb. In Anais do Workshop de Informática na Escola. SBC.

Reis, R., Rodriguez, C., Lyra, K., Isotani, S., Stamato, É., and Gotardo, R. (2016). Experiência de uso de jogos educacionais digitais individuais em contextos de colaboração. In Anais do Workshop de Informática na Escola, volume 22, page 485.

Rodrigues, L., Bonidia, R. P., and Brancher, J. D. (2017). A math educacional computer game using procedural content generation. In Simpósio Brasileiro de Informática na Educação-SBIE, volume 28, page 756.

Silva, R. A., Paula, M. M., and Sarlas, L. M. (2011). Utilização de jogos para pessoas com necessidades educativas especiais: uma análise experimental. In Simpósio Brasileiro de Informática na Educação-SBIE, volume 1.

Susi, T., Johannesson, M., and Backlund, P. (2007). Serious games: An overview.

Weber, K. (2001). Student difficulty in constructing proofs: The need for strategic knowledge. Educational studies in mathematics, 48(1):101-119. 